Journal of Solid State Chemistry 219 (2014) 37-42

\title{
Preparation of resveratrol-loaded nanoporous silica materials with different structures
}

Margarita Popova ${ }^{\text {a,* }}$, Agnes Szegedi ${ }^{\text {b }}$, Vesselina Mavrodinova ${ }^{a}$, Natasa Novak Tušar ${ }^{\text {d }}$, Judith

Mihály $^{\mathrm{b}}$, Szilvia Klébert ${ }^{\mathrm{b}}$, Niko Benbassat ${ }^{\mathrm{c}}$, Krassimira Yoncheva ${ }^{\mathrm{c}}$

${ }^{a}$ Institute of Organic Chemistry with Centre of Phytochemistry, Bulgarian Academy of Sciences, 1113 Sofia, Bulgaria

${ }^{b}$ Research Centre for Natural Sciences, Institute of Materials and Environmental Chemistry, Hungarian Academy of Sciences, 1117 Budapest, Magyar tudósok körútja 2., Hungary

${ }^{c}$ Faculty of Pharmacy, 2 Dunav Str., 1000 Sofia, Bulgaria

${ }^{\mathrm{d}}$ National Institute of Chemistry, Ljubljana, Slovenia

\section{A b s t r a c t}

Solid, nanoporous silica-based spherical mesoporous MCM-41 and KIL-2 with interparticle mesoporosity as well as nanosized zeolite BEA materials differing in morphology and pore size distribution, were used as carriers for the preparation of resveratrol-loaded delivery systems. Two preparation methods have been applied: (i) loading by mixing of resveratrol and mesoporous carrier in solid state and (ii) deposition in ethanol solution. The parent and the resveratrol loaded carriers were characterized by XRD, TEM, N2 physisorption, thermal analysis, and FT-IR spectroscopy. The influence of the support structure on the adsorption capacity and the release kinetics of this poorly soluble compound were investigated. Our results indicated that the chosen nanoporous silica supports are suitable for stabilization of trans-resveratrol and reveal controlled release and ability to protect the supported compound against degradation regardless of loading method. The solid-state dry mixing appears very effective for preparation of drug formulations composed of poorly soluble compound.

\section{Introduction}

Mesoporous silicas and zeolites are promising materials for various aspects of biomedical applications. The preparation of meso - and microporous silica delivery systems for drugs and bioactive molecules is based on the advantages of these materials- tunable pore size, 
controlled particle size and morphology, dual-functional surface (external and internal) and chemical composition [1-3]. Thus, their application as drug carriers can solve some problems associated with low stability and poor bioavailability of the active molecules.

Resveratrol (trans-3,4,5-trihydroxystilbene), $(\mathrm{R})$ is a potential antioxidant with strong antiinflammatory and antiproliferative properties, which can be found in grapes, nuts, fruits and

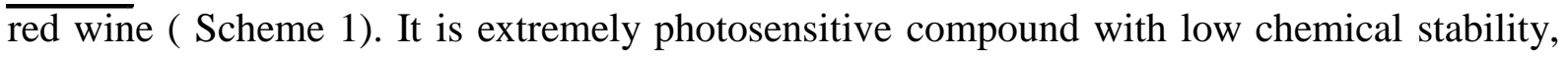
which limits its beneficial therapeutic effects [4]. Thus, loading of resveratrol in nanoporous silica systems can stabilize and protect it from degradation. In addition, the increased specific surface area of mesoporous silica particles could eventually improve its bioavailability.

It is well known that preparation method of drug formulations can influence the solubility of active substance, its loading degree and release profile. Most investigated methods regarding improvement of water solubility are surfactant solubilisation, complexation and micronisation of drugs. A more efficient method to increase drug solubility in water and dissolution velocity was developed at the beginning of the nineties by drug amorphization. A review of some nanotechnologies, commercialized to help deliver poorly water-soluble drugs is made by Kharb et al. [5]. Production of nanocrystalline particles has been attained by different milling techniques leading to amorphization and hence to drug solubility increase. Wet-milling technique [6], physical mixing (paste) [7], ball milling and high pressure homogenization in water [8,9], spray-drying of the drug dissolved in its amorphous/nanocrystalline state on the support [10] and even melt method [11], consisting in heating of a mixture of the drug (itraconazole or ibuprofen) with SBA-15 support have been developed for drug amorphization.

In this study a procedure for dry physical mixing of resveratrol and two types of supports has been applied. Solid nanoporous silica-based materials differing in morphology, topology and pore size distribution, such as mesoporous pure silica (spherical MCM-41 and KIL-2) and nanosized silica-alumina zeolite BEA have been used as carriers. Vibrational ball mill mixer was applied for simultaneous amorphization and loading of the poorly water-soluble drug. To the best of our knowledge, such type of mechanical deposition of resveratrol on the chosen silica nanomaterials has not been reported. This solid-state procedure for encapsulation has two important advantages. Dry mixing ensures partial amorphization of resveratrol crystallites, expected to increase its water solubility. The other priority is that the whole amount of added drug substance should remain on the carrier without any loss at preparation. The physico-chemical characteristics, the adsorption capacity and the drug-release kinetics of the formulations prepared by the solid-state method have been compared to their analogues obtained by the conventional method of encapsulation in solution.
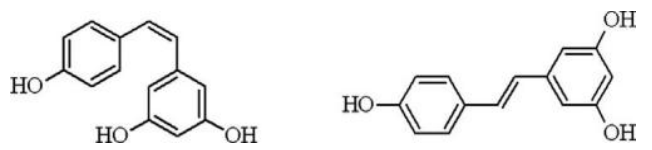

Scheme 1. Cis- and trans-resveratrol 


\section{Experimental}

\subsection{Synthesis of spherical MCM-41 silica material}

MCM-41 with $100 \mathrm{~nm}$ particle size was prepared according to the procedure of Huh et al. [12]. This sol-gel procedure is carried out at $80^{\circ} \mathrm{C}$ without co-solvent, in water solution and applying $\mathrm{NaOH}$ as a catalyst. The relative molar composition of the reaction mixture was: 1 TEOS:0.12 $\mathrm{C}_{16}$ TMABr:0.31 NaOH: $1190 \mathrm{H}_{2} \mathrm{O}$. The formed gel was aged at $80^{\circ} \mathrm{C}$ for $2 \mathrm{~h}$, then washed with distilled water until neutral $\mathrm{pH}$, and dried at ambient. Template removal of MCM-41 materials was carried out in air up to $550^{\circ} \mathrm{C}$ with $1{ }^{\circ} \mathrm{C} / \mathrm{min}$ rate for $5 \mathrm{~h}$.

\subsection{Synthesis of nanosized KIL-2 material}

Mesoporous disordered silicate KIL-2 [13] was prepared by two-step synthesis in molar ratio of 1 TEOS:0.5 TEA:0.1 TEAOH: $11 \mathrm{H}_{2} \mathrm{O}$. In the first step tetraorthosilicate (TEOS $98 \%$, Acros) and triethanolamine (TEA 99\%, Fluka) were stirred for $30 \mathrm{~min}$. Then demineralized water was added to the above mixture, followed by the addition of tetraethyammonium hydroxide (TEAOH 20\%, Acros). The solution was mixed with a magnetic stirrer to obtain a homogeneous gel. The final gel was aged overnight at room temperature and then dried in an oven at $50^{\circ} \mathrm{C}$ for $24 \mathrm{~h}$. In the second step the gel was solvothermally treated in ethanol in a Teflon-lined stainless autoclave at $150^{\circ} \mathrm{C}$ for $48 \mathrm{~h}$. Template was removed by calcination at $500^{\circ} \mathrm{C}$ for $10 \mathrm{~h}$ in air flow.

\subsection{Synthesis of nanosized BEA zeolite}

Zeolite beta nanoparticles were synthesized according to Ref. [14]. In brief, the synthesis procedure includes the preparation of a precursor mixture with the following molar composition: $25.00 \quad \mathrm{SiO}_{2}: 0.25 \mathrm{Al}_{2} \mathrm{O}_{3}: 9.0 \mathrm{R}: 0.35 \mathrm{Na}_{2} \mathrm{O}: 295.0 \mathrm{H}_{2} \mathrm{O}$, where $\mathrm{R}$ is the organic templating agent tetramethylammonium (TEA) (Sigma, 20\% TEAOH in water). The silica source was derived from a colloidal silica suspension Bindzil 30/360 (Eka Nobel, Sweden) containing $31.1 \mathrm{wt} \% \mathrm{SiO}_{2}$ and $0.6 \mathrm{wt} \% \mathrm{Na}_{2} \mathrm{O}$. The suspension was freeze-dried to powder. The aluminum source was aluminum isopropylate (Sigma). The obtained initial mixture was a clear homogeneous solution, which was subjected to hydrothermal treatment at $100^{\circ} \mathrm{C}$ for 9 days. The crystalline nanoparticles were purified by three times centrifugation at 20,000 rpm for $1 \mathrm{~h}$, followed by redispersion in distilled water using an ultrasonic bath to obtain a colloidal sol with a pH of about 9.5. The sample in its H-form, H-BEA, is used for further resveratrol loading and is designated as $\mathrm{R} / \mathrm{BEA}$. 


\subsection{Characterization}

X-ray patterns were recorded by Philips PW 1810/3710 diffractometer with BreggBrentano parafocusing geometry applying monochromatized $\mathrm{Cu} K \alpha \quad(\lambda=0.15418 \mathrm{~nm})$ radiation $(40 \mathrm{kV}, 35 \mathrm{~mA})$ and proportional counter.

Nitrogen physisorption measurements were carried out at $-196{ }^{\circ} \mathrm{C}$ using Quantachrome Autosorb 1C Gas Sorption Instrument. The BET surface area, the mean pore diameter, and the total volume of the samples was calculated and presented in Tables 1 and 2. The pore-size distributions were calculated from the desorption branch of the isotherms with the $\mathrm{BJH}$ method. Samples were pre-treated at $80^{\circ} \mathrm{C}$ before measurements.

Thermogravimetric measurements (TG) were performed with a Setaram TG92 micro balance with a heating rate of $5^{\circ} \mathrm{C} / \mathrm{min}$ in air flow.

Attenuated total reflection infrared (ATR-FTIR) spectra were recorded by means of a Varian Scimitar 2000 FT-IR spectrometer equipped with a MCT (mercury-cadmium-tellur) detector and a single reflection ATR unit (SPECAC "Golden Gate") with diamond ATR element. In general, 128 scans and $4 \mathrm{~cm}^{-1}$ resolution was applied. For all spectra ATRcorrection was performed (Varian ResPro 4.0 software).

\subsection{Resveratrol loading and in-vitro release measurements}

The powdered samples were loaded with resveratrol by two different methods: deposition by ethanol solution and by solid-state method. Ethanol was chosen as a solvent for drug loading because of high solubility of $(\mathrm{R})$ in it. Deposition of resveratrol in solution was carried out by soaking the silica carriers, under continuous magnetic stirring for $24 \mathrm{~h}$ at $37^{\circ} \mathrm{C}$ in ethanolic solution of resveratrol. In practice $100 \mathrm{mg}$ of resveratrol was dissolved in $5 \mathrm{ml}$ of abs. ethanol and $100 \mathrm{mg}$ of the carrier dried at $120^{\circ} \mathrm{C}$ was put into this solution. Than $20 \mathrm{ml}$ of water was added to the solution and the ethanol was evaporated, the resveratrol loaded samples were recovered by centrifugation and dried for $24 \mathrm{~h}$ at $40^{\circ} \mathrm{C}$. These preparations are designated as R/MCM-41(S), R/BEA(S) and R/KIL-2)(S).

For the solid state method, 1:1 weight ratio of resveratrol to mesoporous carrier was used. Resveratrol was mixed with the carriers at room temperature by high energy milling in a DDR-GM 9458 type vibrational ball mill mixer for $3 \mathrm{~min}$. The as-prepared samples are designated as R/MCM-41(SS), R/BEA(SS) and R/KIL-2) (SS).

In-vitro drug release experiments were performed in phosphate buffer $(\mathrm{pH}=.4)$ at $37^{\circ} \mathrm{C}$, under stirring at a rate of $100 \mathrm{~min}^{-1}$. At appropriate time intervals, $3 \mathrm{ml}$ samples were withdrawn and analyzed with UV-vis spectroscopy at a wavelength of $306 \mathrm{~nm}$. 


\section{Results and discussion}

\subsection{Material characterization}

The parent silica nanomaterials as well as their resveratrol-loaded formulations were characterized before and after resveratrol deposition using several techniques.

The low-angle powder XRD patterns of KIL-2 and MCM-41 indicate that KIL-2 has a disordered mesoporous structure, whereas MCM-41 shows an ordered 2D hexagonal (p6mm) pore arrangement ( Fig. 1). The pattern of calcined nanobeta material in its H-form ( Fig. 1) show the typical diffraction peaks of BEA-type topology with the most intensive reflections at 7.6 and $22.412 \theta^{\circ}[15]$.

In Fig. $1 \mathrm{~A}$ the XRD pattern of grinded $\mathrm{R}$ is compared to that of commercial form of the drug. The observed intensity decrease proves that treatment in the vibrational mixer leads to substantial amorphization of the antioxidant, being one of the objectives of solid-state preparation method. The intensity of the reflections of the grinded non-loaded resveratrol is $58 \%$ of the pure one. Presence of crystalline resveratrol phase is registered on all loaded samples regardless of the method of drug loading. The most intensive XRD lines of the drug at $2 \theta^{\circ}=16.4,19,22.3,23.6$, and 28.3 are clearly visible for the solid-state ( Fig. 1A) or solution-loaded ( Fig. 1B) preparations. This is an evidence that resveratrol is not only introduced in the pore channels, but can be found on the outer surface of the small nanoparticles or in the voids among the particles.
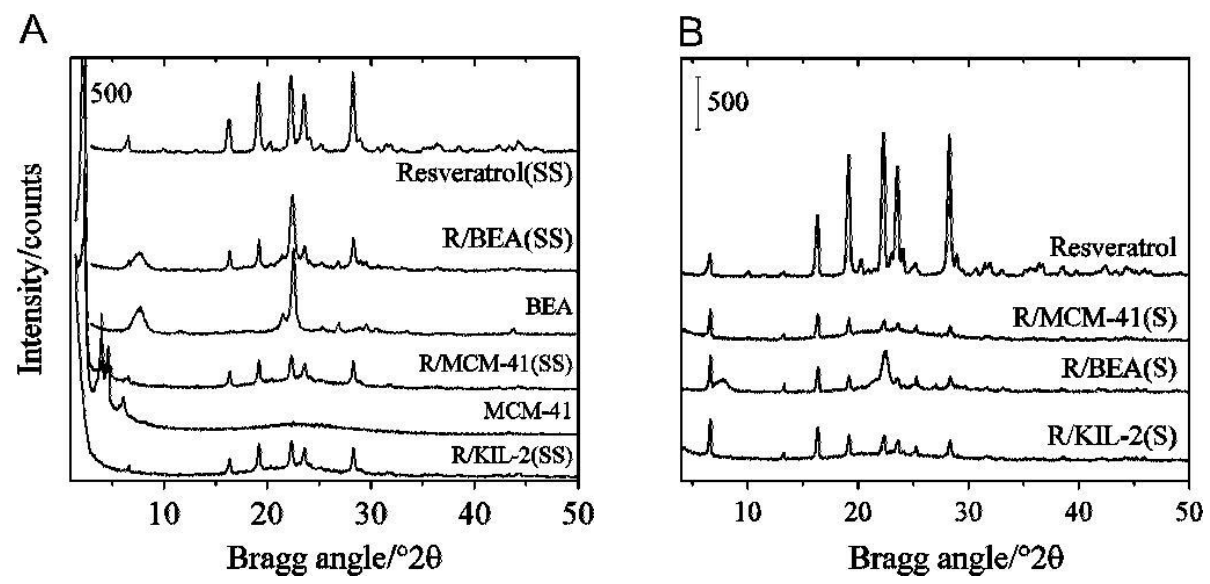

Fig. 1. XRD patterns of resveratrol, zeolite BEA and resveratrol loaded systems prepared by solid-state method (A)and in solution(B). 


\section{Table 1}

Textural and physico-chemical properties of the parent KIL-2 and MCM-41 mesoporous silica materials and their resveratrol-loaded formulations.

\begin{tabular}{|c|c|c|c|c|}
\hline Samples & $\begin{array}{l}\text { BET } \\
\left(\mathrm{m}^{2} / \mathrm{g}\right)\end{array}$ & $\begin{array}{l}\text { Pore diameter } \\
(\mathrm{nm})\end{array}$ & $\begin{array}{l}\text { Pore volume } \\
\left(\mathrm{cm}^{3} / \mathrm{g}\right)\end{array}$ & $\begin{array}{l}\text { Loaded resveratrol } \\
(\mathrm{wt} \%)^{*}\end{array}$ \\
\hline MCM-41 & 1175 & 2.7 & 0.97 & - \\
\hline R/MCM-41(S) & 320 & 2.5 & 0.26 & 39.8 \\
\hline R/MCM-41(SS) & 306 & 2.5 & 0.27 & 46.6 \\
\hline KIL-2 & 664 & 15.2 & 1.28 & - \\
\hline R/KIL-2(S) & 320 & 9.8 & 0.72 & 35.8 \\
\hline R/KIL-2(SS) & 232 & 10 & 0.52 & 48.3 \\
\hline
\end{tabular}

* Determined by TGA by heating from $180^{\circ} \mathrm{C}$ to $600^{\circ} \mathrm{C}$.

\section{Table 2}

Textural and physico-chemical properties of the parent BEA zeolite and its resveratrol-loaded formulations.

\begin{tabular}{llllll}
\hline & $\begin{array}{l}\text { BET } \\
\left(\mathrm{m}^{2} / \mathrm{g}\right)\end{array}$ & $\begin{array}{l}\text { Total pore } \\
\text { volume } \\
\left(\mathrm{cm}^{3} / \mathrm{g}\right)\end{array}$ & $\begin{array}{l}\text { Mesopore } \\
\text { volume } \\
\left(\mathrm{cm}^{3} / \mathrm{g}\right)\end{array}$ & $\begin{array}{l}\text { Micropore } \\
\text { volume } \\
\left(\mathrm{cm}^{3} / \mathrm{g}\right)\end{array}$ & $\begin{array}{l}\text { Loaded } \\
\text { resveratrol } \\
(\mathrm{wt} \%)^{*}\end{array}$ \\
\hline BEA & 761 & 0.46 & 0.34 & 0.12 & - \\
R/BEA(S) & 34 & - & n.d. & - & 48.4 \\
R/BEA(SS) & 18 & 0.04 & n.d. & - & 49.2 \\
\hline
\end{tabular}

$*$ Determined by TGA by heating from $180^{\circ} \mathrm{C}$ to $600^{\circ} \mathrm{C}$.

According to SEM analysis (not shown), the size of the Beta zeolite crystallites is about 40$50 \mathrm{~nm}$. Images show that the particles stick together to $100-150 \mathrm{~nm}$ agglomerates and mesopores are formed among them.

The particle size of the pure resveratrol and the resveratrol loaded samples was calculated according to the Sherrer equation and it is $35 \mathrm{~nm}$. Resveratrol loading resulted in higher intensity decreases than the expected 50\% (32\% for R/MCM-41(SS), 40\% for R/BEA(SS) and $37 \%$ for R/KIL-2(SS)). If we compare the intensity of resveratrol loaded by solution method to pure resveratrol we can find about $10 \%$ resveratrol. Comparing these data with the loaded amount determined by TG analysis we can conclude that a significant part of resveratrol is in amorphous form, not detectable by XRPD.

Nitrogen adsorption and desorption isotherms of the parent, and resveratrol loaded MCM41, Beta, and KIL-2 samples are presented in Fig. 2. The calculated textural parameters of MCM-41 and KIL-2 supports are summarized in Table 1, and for BEA in Table 2. The isotherms of MCM-41 samples are reversible and do not show any hysteresis loop ( Fig. 2A). They exhibit a sharp increase at a relative pressure between $\mathrm{p} / \mathrm{p}_{0=0} 0.2-0.4$, which is associated with capillary condensation of nitrogen in the meso-pores and also indicates a narrow pore 
A

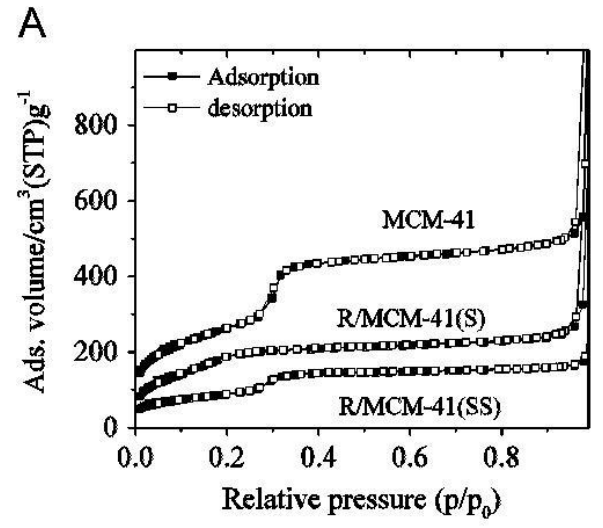

C

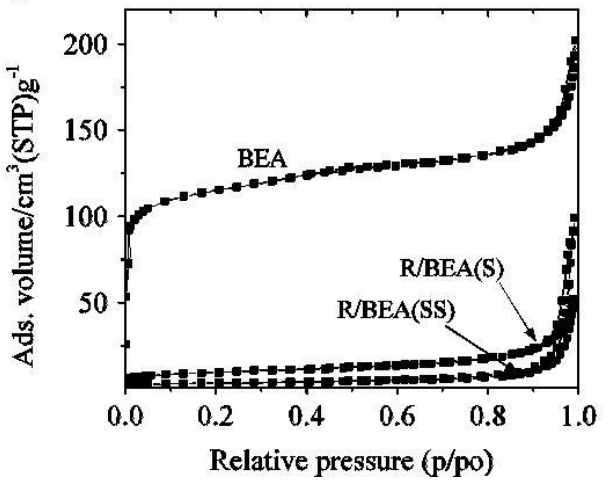

Fig. 2. $\mathrm{N}_{2}$ adsorption-desorption isotherms of the parent and the resveratrol-loaded MCM-41 (A), KIL-2 (B) and zeolite BEA (C) prepared by solid-state (SS) method and in solution (S).

size distribution. In isotherm of KIL-2 ( Fig. 2B), the hysteresis loop appears on the parent and the loaded materials at much higher relative pressure, which is an indication for the presence of bigger mesopores and high textural mesoporosity (see also Table 1). A sharp increase, typical for the micropore filling in zeolites at low relative pressures can be observed on the parent nano BEA sample. Then, at higher pressures, a hysteresis loop indicating intraparticle mesoporosity among the agglomerated zeolite nanoparticles appears ( Fig. 2C).

The textural parameters after $\mathrm{R}$ deposition alter significantly. The observed decrease in the surface area and pore volume is indicative for partial pore filling in the case of MCM-41 and KIL-2 after the solid-state loading of the drug. The change of I. type isotherm for BEA zeolite support is an indication for micropore filling or rather plugging. The microporosity of this material with much narrower channel system actually disappears and the total pore volume is strongly reduced ( Table 2). For both mesoporous supports loaded with $\mathrm{R}$ in ethanol solution, also partial pore filling is observed.

ATR FT-IR method was used to investigate the interaction between resveratrol molecule and the nanoporous carriers after its deposition ( Fig. 3). The FT-IR spectrum of R shows three characteristic strong bands at 1606,1585 and $1384 \mathrm{~cm}^{-1}$, belonging to double bond $\mathrm{C}-\mathrm{C}$ stretchings of aromatic and olefinic groups, and of ring $\mathrm{C}-\mathrm{C}$ stretching, 
A

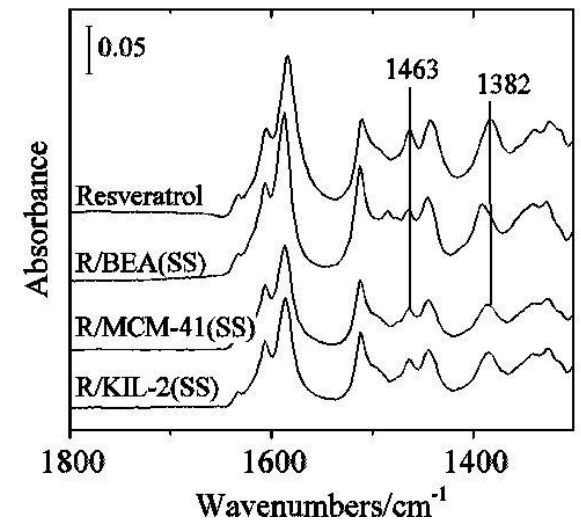

B

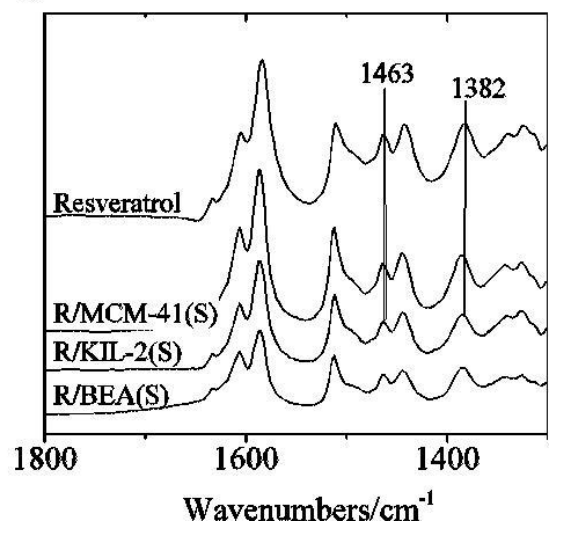

Fig. 3. ATR FT-IR spectra of resveratrol, and the resveratrol-loaded silica samples prepared by solid-state method (A) and in solution (B).

respectively. The bands at 1511,1443 and $1324 \mathrm{~cm}^{-1}$ can be assigned to in plan and out of plane $\mathrm{C}-\mathrm{H}$ bending, respectively [16]. After encapsulation into silica/zeolite framework, a slight blueshift of the resveratrol IR bands corresponding to vinylidene group and a decrease in intensity of the vinylidene $\mathrm{C}-\mathrm{H}$ in plane bend $\left(1324 \mathrm{~cm}^{-1}\right)$ can be observed. These spectral features due to perturbation at vinylidene dipole moments suggest an interaction of resveratrol $\mathrm{C}=\mathrm{C}$ with the silica/zeolite framework. The most pronounced changes are witnessed in case of BEA zeolite (solid-state preparation; shift of 1384 to $1391 \mathrm{~cm}^{-1}$ of $\mathrm{C}-\mathrm{C}$ ring stretching) indicate a nanoconfinement of resveratrol in the micropores/mesopores of BEA. For all cases, the out of plane $\mathrm{C}-\mathrm{H}$ bending of $\mathrm{RCH}=\mathrm{CHR}\left(965 \mathrm{~cm}^{-1}\right.$, not shown), diagnostic for transconfiguration around the di-substituted double bond [17], remains unchanged, indicating that the trans-configuration is preserved during encapsulation.

\subsection{Resveratrol loading and in vitro release}

The adsorbed amount of $\mathrm{Rl}$ was evaluated by thermogravimetric analysis (TGA) considering the weight loss at heating the resveratrol-loaded preparations. At temperatures up to $180^{\circ} \mathrm{C}$ the formulations lose the physisorbed water ( Fig. 4). Above this temperature, the onset of short temperature interval of melting of the supported substance follows, and then fast drug decomposition occurs. The value for the $\mathrm{R}$ loading is listed in Tables 1 and 2. It is calculated from the TGA data by subtracting the weight loss due to water released from room temperature to $180^{\circ} \mathrm{C}$, from the total weight loss at $600^{\circ} \mathrm{C}$. With the exception of the mesoporous silicates loaded by ethanol solution, almost complete retention of the deposited compound, corresponding to the envisaged 1:1 resveratrol-carrier weight ratio 

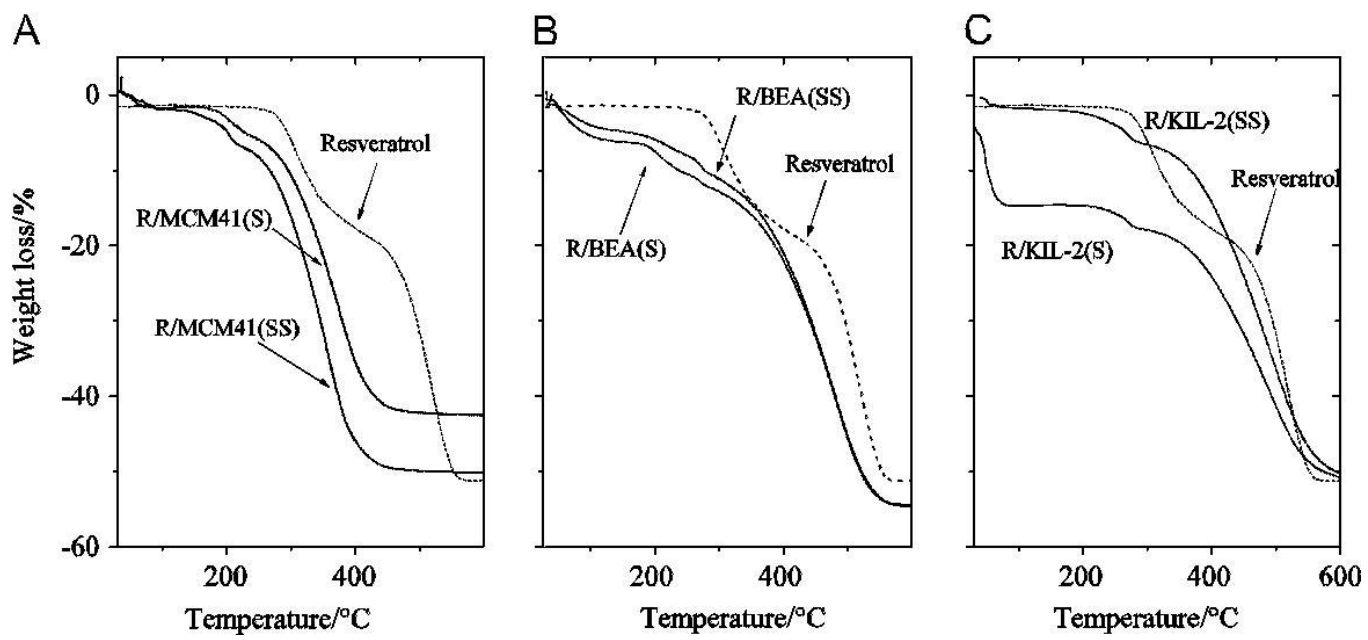

Fig. 4. TG profiles of resveratrol-loaded MCM-41 (A), BEA (B) and KIL-2 (C) in solid-state (SS) and in solution (S).
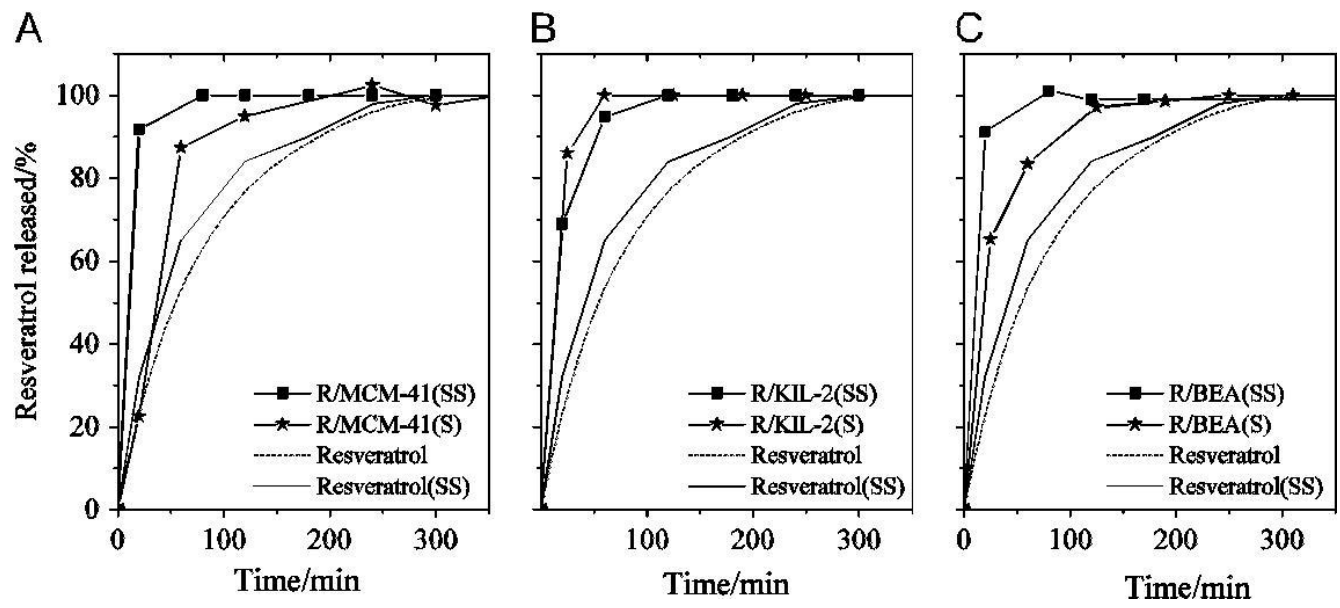

Fig. 5. Release profiles of unsupported resveratrol, and the resveratrol-loaded MCM-41 (A), KIL-2 (B) and BEA (C) drug delivery systems prepared in solid state and in solution.

is registered for the tree studied carriers ( Tables 1 and 2). This result points to the high effectiveness of the solid-state procedure for preparation of drug delivery systems. As it was expected and the TGA determinations showed, almost the whole amount of added sub-stance has remained on the carrier and evolved upon heating up to $600^{\circ} \mathrm{C}$. The lower adsorption capacity for both R/MCM-41(S) and R/ KIL-2(S) preparations in comparison to the BEA zeolite can be explained by the difference in their pore size distribution. The much larger size of the mesoporous channels and, respectively, their higher pore volume, suggest more facile diffusion of resveratrol out of the channel system, when the loading is carried out in solution. 
Resveratrol release was carried out in phosphate buffer at $\mathrm{pH}=7.4$ and the kinetics of its evolution were followed by UV-vis spectroscopy at a wavelength of $306 \mathrm{~nm}$, the characteristic signal of the bioactive trans-resveratrol form. The data showed only this signal in the samples taken from the released solution. The release experiments were repeated 3 times with a period of one week between them and only trans-resveratrol was detected. This result is an indication that the loading on the chosen nanoporous carriers prevents the transformation of the supported bioactive trans-form to the less active cis-form. Thus, deposition of $\mathrm{R}$ on powdered silica-based carriers with mesoporous texture resulted in its stabilization and enhanced solubility.

The release profiles of the loaded supports are presented in Fig. 5. Total release in about three hours was achieved for all resveratrol-loaded samples regardless of the preparation method. On the contrary, the free resveratrol and even the resveratrol treated by milling but not loaded on the carriers dissolves more slowly compared to that loaded in nanoporous systems. It indicates that the selected carriers and loading procedures substantially facilitate its dissolution. For the KIL-2 support ( Fig. 5B) almost similar release profiles are observed for both preparation methods. This parity can be explained by the presence of much larger mesopores, vis. the empty voids with the highest adsorption volume ( Table 1), formed among the nanoparticles of the KIL-2 carrier. This specific pore texture presumes equivalent location and surrounding of the supported substance and analogous adsorption characteristics.

In the case of MCM-41 ( Fig. 5A) and the zeolite BEA ( Fig. 5C), the solid state method of loading ensures faster release compared to the analogue formulations prepared in ethanol solution. The presence of multiple pore system (internal and external mesoporosity in MCM41 , and a simultaneous presence of micro- and mesopores in nanosized zeolite) presumes selective access of resveratrol to the pore system of the supports. This can result in diversity in the release kinetics depending on the method used for loading. The solid-state deposition occurs, most probably, on the outer surface of both supports and ensures faster and close release kinetics. The presence of narrower pore channels in the zeolite carrier is the reason for the lower rate of R delivery from it, compared to MCM-41, when the drug is loaded by the liquid deposition method. Resveratrol amorphization during the grinding procedure upon dry mixing also contributes to the easier release when the substance is loaded in solid-state.

\section{Conclusions}

Loading of resveratrol on nanoporous MCM-41, KIL-2 silicas, and on nanosized BEA zeolite by two different methods (in solid-state and from ethanol solution) resulted in similar high loading capacity of the antioxidant. Considering the release profiles, all preparations showed better resveratrol solubility compared to the free resveratrol, regardless of the support structure and the method of loading. In vitro release process at $\mathrm{pH}=7.4$ showed faster resveratrol delivery from MCM-41 and BEA carriers pre-pared by the solid state method in comparison to that in ethanol solution. Resveratrol release from both KIL-2 preparations was equally fast independently of the loading method. This effect can be associated with the 
different pore texture of the carriers. The applied solid-state procedure of preparation appears very effective for loading of the medical compound. It ensures preservation of the whole loaded drug amount as well as improves significantly the solubility of the loaded substance due to amorphization upon grinding. Deposition into the powdered mesoporous supports stabilizes the bioactive trans-resveratrol form.

\section{Acknowledgment}

The authors wish to acknowledge Dr. Valentin Valtchev for the disposition of the nano BEA zeolite and for its $\mathrm{N}_{2}$ physisorption characterization. Support for this work in the framework of the Hungarian-Bulgarian Inter-Academic Exchange Agreement is gratefully acknowledged.

\section{References}

[1] M. Vallet-Regi, A. Ramila, R.P del Real, J. Perez-Pariente, Chem. Mater. 13 (2001) 308311.

[2] Sh. Wang, Microporous Mesoporous Mater. 117 (2009) 1-9.

[3] P. Horcajada, C. Marquez-Alvarez, A. Ramila, J. Perez-Pariente, M. Vallet-Regi, SolidState Sci. 8 (2006) 1459-1465.

[4] E.H. Gokce, E. Korkmaz, E. Dellera, G. Sandri, M.C. Bonferoni, O. Ozer, Int. J. Nanomed. 7 (2012) 1841-1850.

[5] V. Kharb, M. Bhatia, H. Dureja, D. Kaushik, Pharm. Technol. 30 (2006) 82-92.

[6] E. Merisko-Liversidge, G.G. Liversidge, E.R. Cooper, Eur. J. Pharm. Sci. 18 (2003) 113120 (Surface modified drug nanoparticles, US Patent 5,145 (1992) 684.).

[7] D.G. Fatouros, D. Douroumis, V. Nikolakis, S. Ntais, A.M. Moschovi, V. Trivedi,

B. Khima, M. Roldo, H. Nazar, P.A. Cox, J. Mater. Chem. 21 (2011) 7789-7794.

[8] C. Keck, S. Kobierski, R. Mauludin, R. Muller, Dosis 24 (2008) 124-128.

[9] C.W. Pouton, Eur. J. Pharm. Sci. 29 (2006) 278-287.

[10] S.-C. Shen, W. Ng, L. Chia, J. Hu, R. Tan, Int. J. Pharm. 410 (2011) 188-195.

[11] R. Mellaerts, J. Jammaer, M. van Speybroeck, H. Chen, J. van Humbeeck,

P. Augustijns, G. van den Mooter, J. Martens, Langmuir 24 (2008) 8651-8659. 
[12] S. Huh, J. Wiench, J.-Ch Yoo, M. Pruski, V. S.-Y. Lin, Chem. Mater. 15 (2003) 4247.

[13] N. Novak Tušar, A. Ristić, G. Mali, M. Mazaj, I Arčon, D. Arčon, V. Kaučič,

N. Zabukovec Logar, Chem. Eur. J 16 (2010) 5783-5793.

[14] M.L. Landau, D. Tavor, O. Regev, M.L. Kalija, M. Herskowitz, V Valtchev,

S. Mintova, Chem. Mater. 11 (1999) 2030-2037.

[15] A- J. Perez-Pariente, J.A. Martens, P.A. Jacobs, Appl. Catal 31 (1987) 35-64.

[16] F. Billes, I. Mohammed-Ziegler, H. Mikosch, E. Tyihák, Spectrochim. Acta, A: Mol. Biomol. Spectrosc. 68 (2007) 669-679.

[17] V. Bertacche, N. Lorenzi, D. Nava, E. Pini, C. Sinico, J. Inclusion Phenom. Macrocyclic Chem. 55 (2006) 279-287. 